\title{
Consistency Management in the Presence of Simultaneous Client-Specific Views
}

\author{
Eddy Truyen, Wouter Joosen, Pierre Verbaeten \\ DistriNet, Dept. Computer Science \\ K.U.Leuven \\ Celestijnenlaan 200A \\ 3001 Leuven, Belgium \\ +32 (0) 16327602 \\ \{eddy, wouter, pv\}@cs.kuleuven.ac.be
}

\begin{abstract}
This paper is about client-specific customization of systems that implement an on-line Internet service in the presence of simultaneous client-specific views. The problem is that each client must be able to customize the running system for use in its own context, without impacting the service behavior that is delivered to other clients. To solve this, we propose to customize the system on a per client request basis, where the system itself consists of a stable core and several extensions that are plugged into the core as needed. However, this approach brings on its own several consistency management problems that must be dealt with in order to make the approach viable. We give an overview of these problems and present a management architecture that deals with these problems.
\end{abstract}

\section{Introduction}

With the Internet and the World Wide Web (WWW) a new trend has come up: on-line distributed services that are remotely accessible from everywhere in the world. There is a multitude of examples: e-commerce, reservation systems, banking systems, dating services, compose your own garden and we come build it for you, etc. Furthermore, the general acceptance of web service technology as supporting platform for enterprise application integration has significantly contributed to this trend: a growing number of enterprises are turning their internal business solutions into on-line services. This allows the development of new services by dynamically composing the existing portfolio of services.

This paper is about customization of the (objectoriented) systems implementing an online service. With customization we mean that the functionality of the system can be easily adapted to client-specific needs. There is a huge market benefit in highly customizable services (e.g [27] gives convincing arguments for this in the context of financial services). As such, for on-line services, the complexity of software has definitely shifted
"construction" to "evolution" [1].

The character of the Internet introduces however a research problem concerning customization of on-line services: a running system instance (the program of the system in execution) is at the same time used by hundreds or even thousands of different client systems, where each client system may have different - possibly conflicting customization needs with respect to the functionality of the service. As a consequence, each client of the system instance must be able to customize the system instance for use in its own context, without affecting the service behavior that is delivered to other clients. We call this problem system customization in the presence of simultaneous client-specific views.

A naïve solution to solve this problem would be to maintain a pool of 'clones' of the same system instance, where all the clones perform actions on common data, but where each clone's implementation is customized to the needs of a specific client. As such, there would be for every client a separate clone of the system instance. In it most simple form, this solution resembles the architecture of Enterprise Java Beans (EJB) where each client (session) is served by a separate session bean instance. Although session beans have been introduced for other purposes, nothing would prevent us from building an EJB server that maintains a pool of session beans, where each session bean is customized to the needs of a specific client session. However, this approach does not scale.

- The concept of state full client session is harder to maintain for Internet applications. Keeping state information at the server for client sessions and user profiles may be infeasible given the potentially large number of simultaneous clients [8].

- $\equiv$ ther problem is that one has to create for every .e a separate code base, which is extremely difficult to maintain in the presence of frequent software updates. For example, assuming that clones are customizations of a common base of existing code, 
software updates of the base due to removal of little bugs will be extremely tedious and therefore does not scale with an increasing number of client-specific views.

When system instances cannot be cloned, we are faced with the problem to change their behavior solely by adding extensions. This leads us to the use of wrappers [6]. The wrapper-based approach enables unanticipated, run-time, instance-level customization, joint use of different extensions (either by disjunctive or conjunctive wrapping) and easy modeling of components that present different interfaces to different clients [13].

Based on this, we describe the customization process of a system implementing a distributed service as follows. We start out with a core system whose functionality is 'stable' in the sense that it is invariant for all clients. Finding the stable core is not easy and requires a lot of knowledge of the application domain. However we suppose that a stable core system can be found. Furthermore there is a set of extensions that each implements an additional feature or new business rule to the core. $\equiv$

Then we present the cust as a run-time and selective combination of extensions to the stable core on a per client request basis. As such, each client $\equiv$ uest enforces a by-request customization of the syste $\square$ here the system itself consists of a stable core and several extensions that are plugged into the core as needed. Which extensions are integrated in the core depends on the client that is currently making the request.

We have already presented elsewhere [26] the Lasagne component model that supports a run-time and selective combination of extensions on per client request basis.

\subsection{Problem statement}

Although a by-request customization seems the only possibility to support customization in the presence of simultaneous client-specific views, this approach brings on its own several consistency management problems that must be dealt with in order to make the approach viable. In section 3 of this paper, we discuss the consistency management problems and define a management architecture on top of the Lasagne component model that solves these problems. In section 2 we give an overview of the Lasagne component model explaining only these features that are necessary to understand the issues addressed in this paper. We discuss related work in section 4 and conclude in section 5 .

\section{The Lasagne Model}

In this section we first describe the software engineering goals that are underlying Lasagne. Then we give a short overview of Lasagne.

\subsection{Motivation behind Lasagne}

In current object-oriented systems, making even minor extensions to software systems requires invasive $\equiv$ inge into existing code and is complicated by the lack of $\nabla_{\text {lity. }}$ This makes extensions tedious to program, makes it difficult to ensure consistency and makes it extremely difficult to plug and unplug extensions. Therefore, a prerequisite for the above customization process to work is that software systems have a modular structure such that extensions are compositional with this structure. In this respect, Lasagne is highly inspired by the aspect-oriented programming (AOP) [12] research that works on providing linguistic mechanisms for programming extensions as modular units that would otherwise 'crosscut' through multiple parts of existing code.

The Lasagne model defines a non-invasive, aspectoriented, per-instance, per-collaboration component extension mechanism; the idea behind this mechanism is changing the behavior of the system without changing the code of core components, but instead encapsulating them within wrappers. In turn, wrappers can be encapsulated in other wrappers if needed.

Wrappers are utterly useful for customizing systems that implement an on-line service: an on-line component instance may have during its lifetime several remote clients, each with different customization needs; with wrappers each client-specific customization simply corresponds to another wrapper around the component instance.

Since wrappers operate at the instance-level, integration of extensions into a running system instance becomes feasible. This allows that the behavior of the system can be adapted to accommodate dynamically evolving customization needs without shutting down the system. For certain application domains, such as the banking and telecommunication sector, this is crucial because these applications need to provide a 7x24h availability.

Wrappers support aspect-oriented programming. A crosscutting extension can be implemented in a modular way as a set of wrappers that work together at multiple core components. Each wrapper can be integrated around its respective core component without requiring invasive change in the code of the core component.

Selection of extensions happens per client request. Since, a client request typically triggers a collaboration (a graph of messages) between multiple component instances of the core system, special attention must be paid on maintaining consistency: $\equiv$ an extension is selected, this decision must be coordir $D$, such that the wrappers of that extension are dynamically plugged in at the appropriate execution points of this collaboration.

The usefulness of wrappers in class-based objectoriented programming languages is however limited by the underlying object model. The most important problem is 
that wrappers cannot be transparently interposed between two component instances due to object identity problems [10]. This leads to severe scalability and consistency problems when using wrappers for dynamic and selective combination of extensions [26].

\subsection{An overview of Lasagne}

Lasagne defines a wrapper programming model and associated component model that realizes the above software engineering goals without suffering the above mentioned object identity problems. It can be implemented on top of any programming language or middleware platform with an open implementation.

We explain Lasagne by an example. Consider a distributed dating system, which manages a set of electronic agendas on behalf of a set of clients. The system implementation consists of two components as shown in Figure 1. The system only implements a stable core.

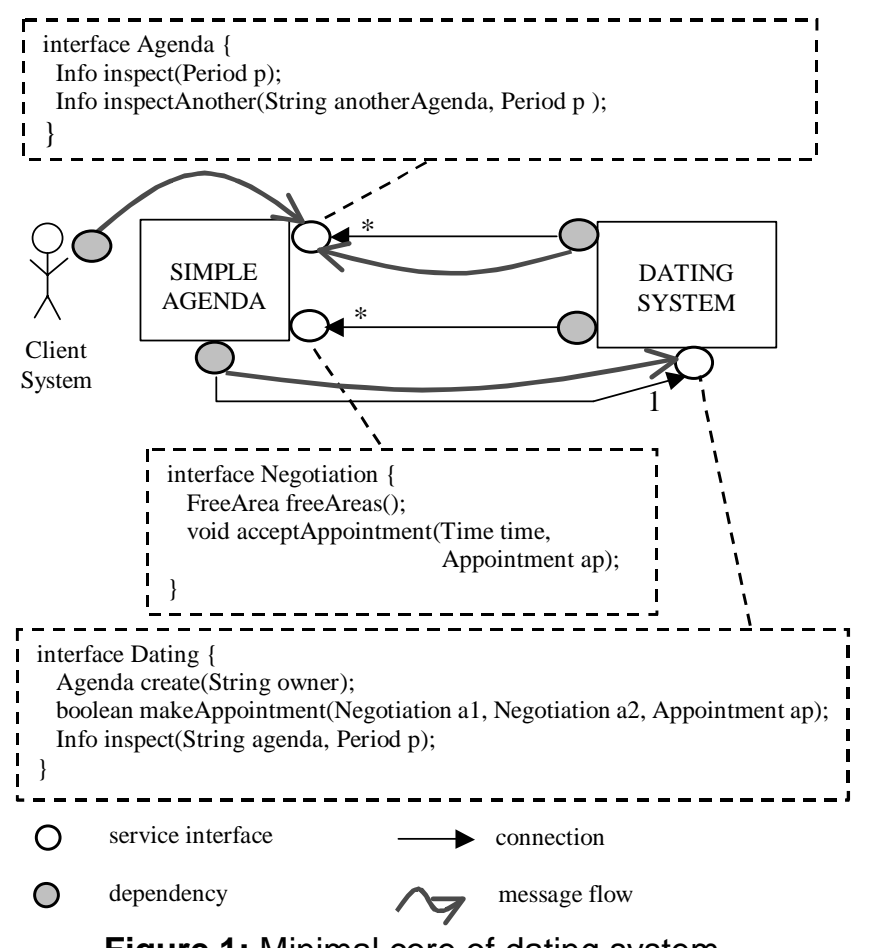

Figure 1: Minimal core of dating system

A component instance in the core system participates in one or more collaborations. For example the curly arrows in Figure 1 show the collaboration for the client request "inspect another agenda". A second collaboration "make appointment" (not shown in Figure 1) consists of the dating system component instance, which coordinates the creation of an appointment between two agenda instances by searching for a point of time that is marked as free area in both agenda's.
Now, suppose that clients can selectively integrate the following extensions into this core dating system:

- A service for making group appointments between more then two agendas in an atomic fashion. As a consequence, agendas must be extended with atomic commit behavior.

- When making an appointment between agendas different strategies for searching a period of time may apply whether the appointment is meant for business or for leisure. The dating system must therefore be able to dynamically switch between these two different appointment strategies.

- E-mail notification: when making a new appointment, some clients may want to send an appropriate e-mail to all the person involved.

- Access control: Some clients want to control the access to their agenda, because they don't want that everybody can inspect or make appointments in their agenda.

To realize dynamic and selective combination of extensions, Lasagne introduces the following concepts and mechanisms:

First, we observe that composition is ideally specified in terms of extensions instead of wrappers, which represent too fine-grained entities. It is really the extension as a whole that clients want to select or unselect for their collaborations with the core system. Therefore, we introduce the notion of an extension identifier, which is an interpretable, high-level name uniquely identifying the extension. Wrapper definitions are specified to be member of an extension by declarative binding to the unique extension identifier. Extension identifiers should be managed in a hierarchical namespace. For the sake of simplicity however we introduce for the above extensions the dummy extension identifiers "group", "leisure", "business", "access" and "email"

Second, a composition policy of a client request is the subset of extensions that must be applied for that client request. The composition policy is externalized from the code of the system implementation, such that it can be controlled in one place: a composition policy is specified as a set of extension identifiers and travels as meta-data with the message flow of its collaboration: it is automatically propagated through the system as the execution of its collaboration advances. As such, the decision which extensions to use for a specific client request travels as a first-class entity together with the message flow of the collaboration, rather then being locked up and scattered across the implementation of the core system.

Third, a composition policy is incrementally defined at run-time by some interceptors. An interceptor intercepts incoming or outgoing messages of a specific component instance and may update their associated composition policy by attaching/discarding extension identifiers. 
Interceptors typically implement a client-specific strategy Interceptors allow expressing that an extension should be executed when a programmer-defined expression is true. Due to the propagating nature of composition policies, an interceptor encapsulates a client-specific customization of an entire collaboration with the guarantee of system-wide consistency.

Lasagne employs a dynamic wrapper mechanism that dynamically composes wrappers into a wrapper chain on a per message basis. To realize this dynamic chain construction a special method dispatch mechanism, called variation point, must be implemented by the underlying middleware platform or programming language When a method of a component instance is called, the variation intercepts this message, interprets the composition policy of the message and will only redirect this message through those wrapper instances whose extension identifier is listed in the composition policy. The reader is deferred to [25] for implementation details of the variation point construct.

\subsubsection{The extension programming model}

Figure 2 shows some of the example extension as integrated in the core dating system. We implement each extension as a coherent module of mixin-like wrappers that work together (e.g. at multiple core components, at client and server side, etc.) to provide a refined or new core service.

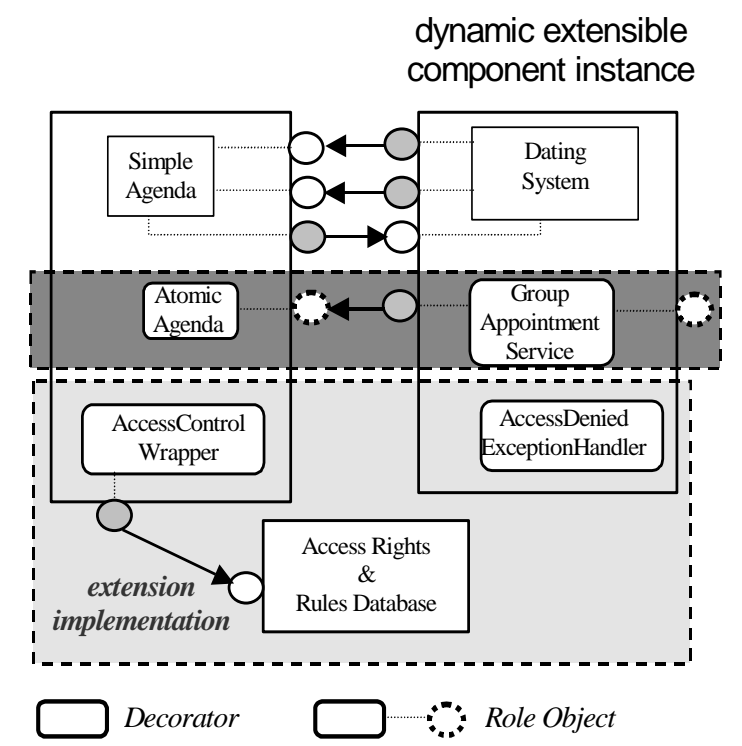

Figure 2 Some of the extensions integrated

A wrapper can specialize a core component by reusing its state, by adding new state components and by constraining the set of behaviors of its operations. To do this the wrapper can performs additional actions before or after forwarding to a dynamically bound inner component. This is similar to the "Decorator" design pattern[6].

However, the inner component is denoted by a special keyword "inner" (see code example of the group appointment extension below). The inner parameter of a wrapper is bound at run-time, somewhat similar to the "super" parameter of mixin-based inheritance, which is bound at class composition time [4]. It is exactly this feature of the wrapper programming model that corresponds with the dynamic construction wrapper chains by the variation point.

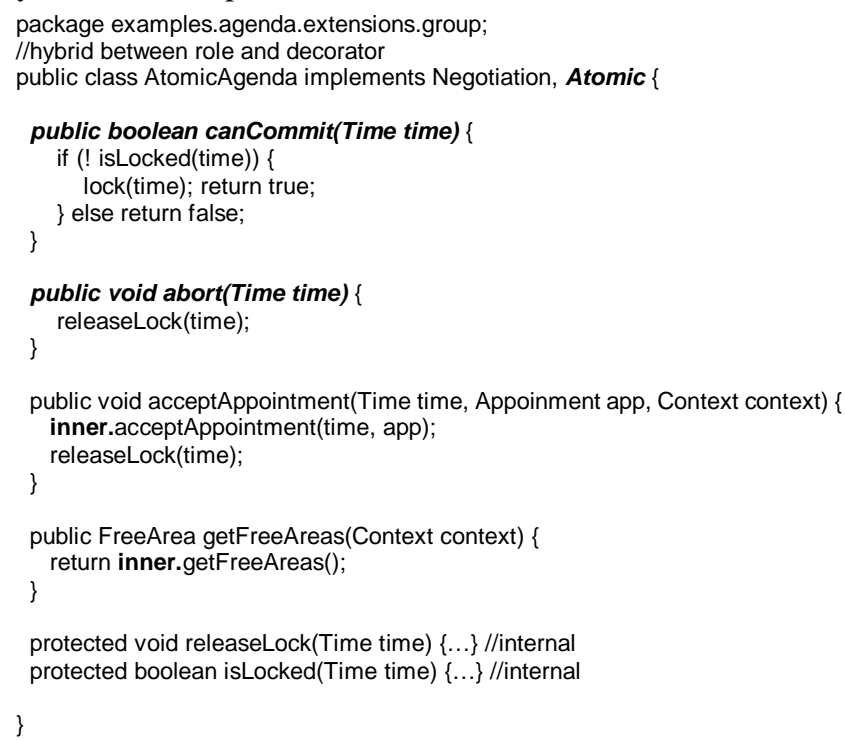

A wrapper can also extend the interface of a core component. In this case the state of the core component is unchanged, but the wrapper can include new operations not found in the parent and new parameters to operations that exists at the parent. This is similar to the "Role Object" design pattern [3]. Outside instances that want to use the newly added interface need to have a reference to the role instance. The Role Object design pattern solves this by passing a specification object to the core component instance and the role instance that matches the specification is returned [3]. Lasagne adopts the same mechanism but passes naturally the extension identifier of the wrapper instance as specification object by means of a special language primitive getRole().

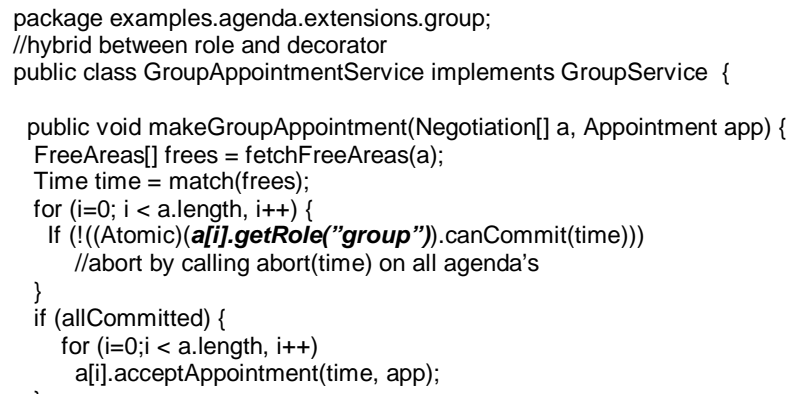


As such, the wrapper programming model of Lasagne combines both the Decorator and the Role Object design pattern. However, the relatively complex object structures required for building up decorators and role objects are not apparent anymore in the programming model of Lasagne, by having shifted complexity from programmer to language implementation.

\subsubsection{Defining client-specific composition policies with interceptors}

As explained above, an interceptor intercepts collaborations and updates their attached composition policy in a context-specific fashion.

Interceptors are deployed around appropriate component instances (which are often the client instances and front-ends of the core system). They intercept either outgoing messages from a specific dependency or incoming message on a specific service interface.

Interceptors are programmable entities that have a simple interface for intercepting component interactions of their co-located component instance. Interceptors typically implement a client-specific strategy that determines which extension identifiers are attached to which collaborations.

The underlying code example illustrates a simple implementation of an interceptor that selects the "leisure" appointment strategy extension when the client makes an appointment.

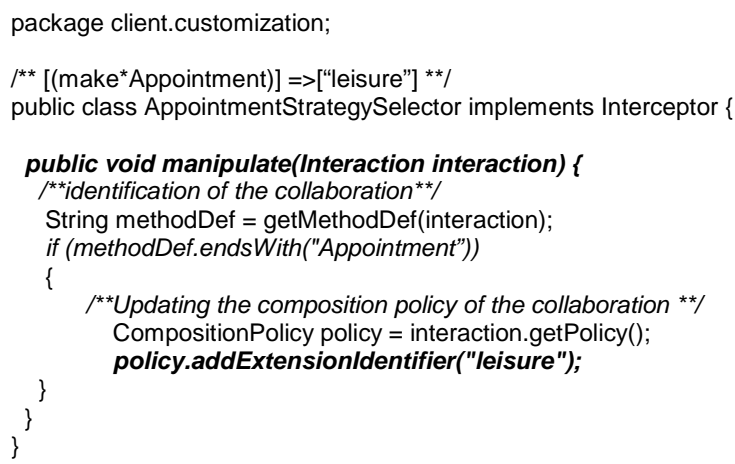

Figure 3 presents a message sequence diagram illustrating a very simple example of the process of clientspecific combination of extensions. In the figure there are two clients. An outgoing interceptor is associated with each client that determines whether the client wants to make a business or a leisure appointment. At the moment depicted by Figure 3 the one client wants to make a leisure appointment, while the other client wants to make a business appointment.

Figure 3 illustrates that once an interceptor has selected an extension for a collaboration, this decision is propagated with the collaboration's message flow such that the wrappers of that extension are plugged in at the appropriate execution points of this collaboration. As such, interceptors encapsulate a client-specific customization of the core system, with the guarantee of system-wide consistency.

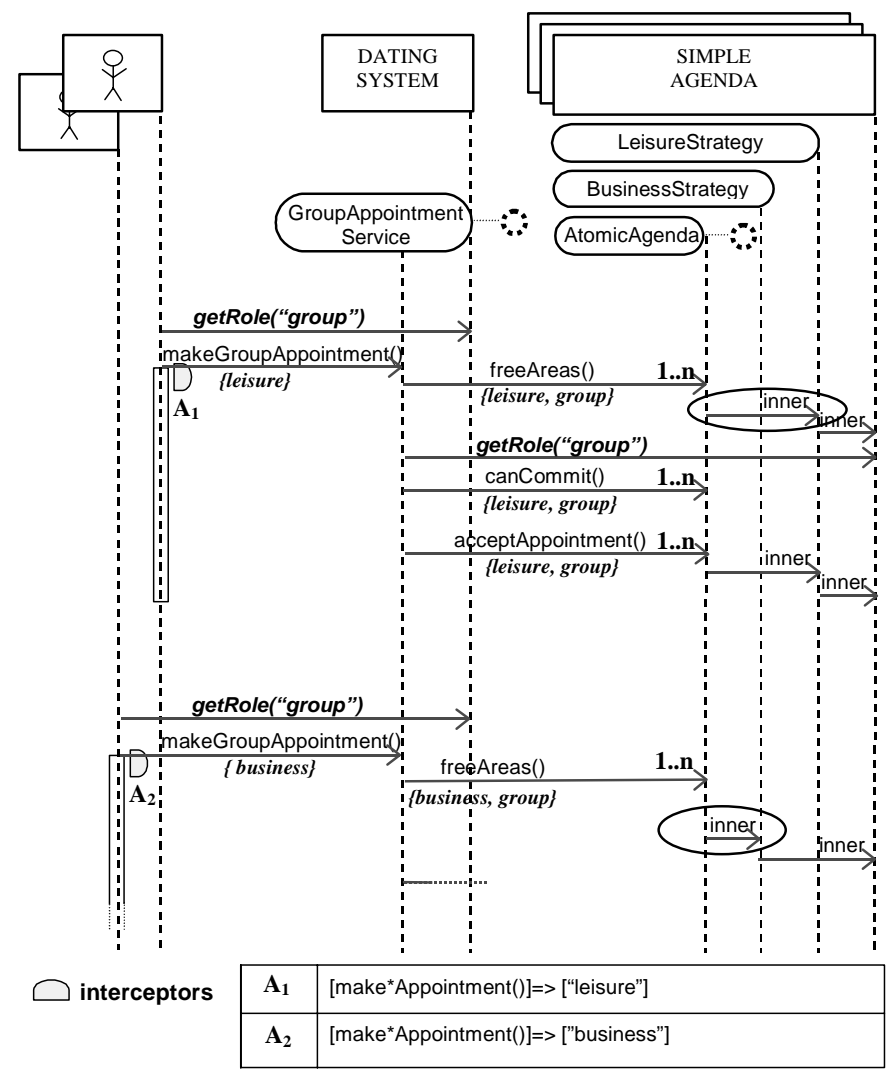

Figure 3 Message Sequence Diagram

Different extensions can of course be combined for the same client request. For example, in Figure 3, both clients want to make a group appointment (however with different appointment strategies). Since the group appointment extension adds role interfaces to the core, references to these roles must be requested via the getRole() primitive. When an operation of the GroupService role is invoked, the Lasagne run-time architecture automatically attaches the extension identifier "group" to the composition policy of the ongoing collaboration, to assure that further execution of the collaboration includes the group appointment extension.

\section{Consistency Management}

A system instance is always running inside a context. With respect to a system that implements an on-line service we distinguish between two kinds of context: the deployment environment in which the system instance is running, and the clients that use the services of that system 
instance. We introduce the role of system manager as the person who installs the system instance in the deployment environment and who makes sure that the system instance stays operational during its lifetime.

To accommodate client-specific customization, the system manager can add extensions on behalf of specific clients. The Lasagne component model allows then to selectively integrate extensions on a per client request basis. However, as stated in the introduction, this approach introduces consistency management problems. We distinguish here between tree kinds of problems:

Malicious clients. A malicious client should not have the power to corrupt a system instance by integrating a erroneous extension.

Run-time integration of new extensions. Consistency problems may possibly arise when a new client-specific extension must be integrated in a running system instance: due to the crosscutting nature of extensions, run-time integration of a new extension requires the wrapping of multiple component instances in an atomic action. As such coordination mechanisms are needed that preserve the consistency of the system while the integration of the extension is in progress.

Inconsistent client-specific composition policies. It is important that clients cannot endanger the integrity of the system by specifying illegal or inconsistent composition policies. We distinguish between two kinds of problems:

- Mandatory extensions: some extensions (e.g. access control) must be imposed on all clients no matter what. Malicious clients should not have a way to get around such mandatory extensions.

- Conflicting extensions: some extensions cannot be applied together for the same client request because they conflict with each other or they just implement the same concern in two different ways (e.g. leisure and business).

It is the responsibility of the system manager to detect such inconsistent client-specific composition policies and deal with them appropriately by either transforming the composition policy to an appropriate one or refusing service to the issuing client.

\subsection{System management architecture}

We now explain from a high-level point of view, a system management architecture that supports integrating new extensions into a running core system instance. This architecture is built on top of the Lasagne wrapper model.

Design philosophy. The basic design philosophy behind the management architecture is that run-time integration of extensions is strictly separated in two consecutive phases:

(1) Deployment of the extensions into the core system. Deployment of an extension means that the extension is being installed in the deployment environment of the system without being "activated". However, after deployment, the extension is ready to be activated and integrated with the core system.

(2) Dynamic selection of extensions on a per client request basis with support from the Lasagne wrapping mechanism to integrate the extensions.

Deployment of extensions is the exclusive privilege of the system manager. As such, clients can only select those extensions that have been deployed before by the system manager. If a client wants to have a new feature added to the system instance, he/she should negotiate this with the system manager.

Deployment of a new extension. We give an overview of the architecture by means of a concrete deployment scenario. Suppose the system manager wants to add a new extension to a running system instance. The system manager makes the new extension first available by uploading the implementation of its wrappers into a global code repository. Each wrapper implementation is named by a code repository identifier that consists of the extension identifier to which that wrapper implementation belongs followed by a '? and the fully qualified name (e.g. class name) of the wrapper implementation.

Secondly there is a configuration management tool that allows the system manager to deploy the new extension into the running system instance. To do this, the configuration management tool must know about all the core component instances running in that system instance. The configuration management tool allows the system manager to specify in an easy way wrapping commands. A wrapping command specifies around which component instances a specific wrapper of the extension must be wrapped. A wrapping command uses code repository identifiers to refer to a specific wrapper implementation. There are three different kinds of wrapping commands. The difference between them lies in the scope at which the wrapping command operates:

- The wrapping command operates at the component type level: a wrapper instance must be deployed around all component instances that implement one or more specific interfaces.

- The wrapping command operates at the component implementation level: a wrapper instance must be deployed around all the instances of a specific component implementation (archive of a set of classes + version number).

- The wrapping command operates at the component instance level: a wrapper instance must be deployed around one or more specific component instances.

Each core component instance is watched over by a local component manager who manages the "wrapper aggregate" of the core component instance and the wrappers that are currently deployed around the core 
instance. A component manager is implemented as a metalevel object that controls the composition logic of how wrappers must be composed around its associated core component instance. For example, when different extensions are deployed around the same component instance, there may be constraints on the order in which their respective wrappers must be applied to a received message. For example, in the above dating system example access control should be performed before the group appointment service. It is not yet clear to us who has the responsibility to state these ordering constraints (the system manager, the extension implementer, or a combination of both), but once this information is stated, the component manager offers this information to the dynamic wrapper mechanism of Lasagne as a partially ordered set of extension identifiers.

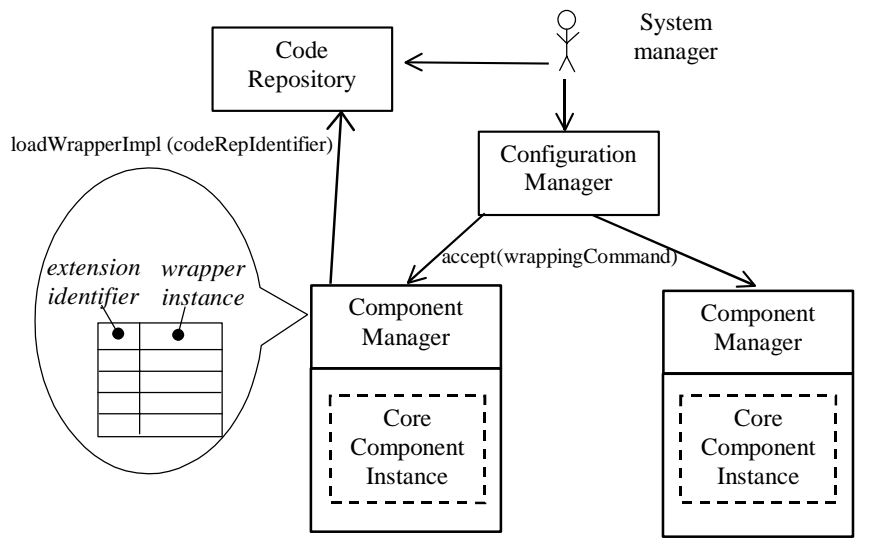

Figure 4 Deployment of extensions

Each component manager has an interface for accepting new wrapping commands from the configuration manager.

The acceptance of a wrapping command results eventually in the construction of a wrapper instance. The system manager can however choose to activate the extension in either a lazy or strict fashion. In a lazy fashion, the construction of the wrapper instances is postponed until a client actually wants to use the extension for the first time. In a strict fashion, the component managers construct the wrapper instances immediately after acceptance of the wrapping commands.

When the wrapper construction is initiated, each component manager loads the wrapper implementations from the code repository by passing the code repository identifier that was specified in the received wrapping command. Once constructed, the wrapper instance is managed in a hash table; the object identity of the wrapper instance is labeled with the extension identifier of that wrapper.

Note that the actual combination and integration of wrappers into the core system is implemented by the dynamic wrapper mechanism of Lasagne.

三loyment-specific interceptors. Besides deploying Dht-specific extensions, the system manager may also want to deploy one or more extensions on his own behalf. For example, he may want to impose a set of specific business rules. Deployment-specific extensions also involve support for non-functional requirements such as security, and reliability.

Of course, the system manager wants to have full control on the composition logic of these deploymentspecific extensions without interference from clients. Lasagne naturally supports this by interceptors that are placed in the deployment environment before the front-ends of the system instance. To make a clear difference, we call these interceptors deployment-specific interceptors, while interceptors at the client side are called client-specific interceptors. Deployment-specific interceptors impose the deployment-specific extensions by attaching the appropriate extension identifiers to the composition policy of every incoming client request. A client-specific interceptor cannot override a depioyment-specific interceptor due to the direction of control transfer from client to system instance. Thus, deployment-specific interceptors have precedence over client-specific interceptors.

A deployment-specific interceptor can of course also be used to dynamically control the composition policy of ongoing collaborations. This allows for example adapting the system instance to changing circumstances in the deployment environment (e.g. a drop in the availability of computational resources) by switching between nonfunctional extensions at run-time. Different extensions can implement the same non-functional requirement, but each extension is optimized for different circumstances in the deployment environment. If the system instance must be adaptable to changing circumstances in the deployment environment, the system manager must deploy the alternative extensions with lazy activation. A deploymentspecific interceptor can then be programmed that monitors the circumstances in the deployment environment and dynamically selects the extension that is best suited to the current circumstances.

\subsection{Dealing with the management problems}

In this section we show how the management architecture deals with the three kinds of consistency management problems.

Malicious clients. After the deployment of a new extension is finished, the system manager publishes the extension identifier (plus a documentation of the feature implemented by this extension) as part of the service interface of the system. As such, clients can only select those extensions that have been deployed before by the system manager. 
Provided that access to the configuration management tool is checked, it becomes more difficult for a malicious client to integrate a corrupting extension into the core system.

Coordination support for integration of new extensions. In a software system that is developed with a traditional programming language, complex coordination protocols are needed to preserve the consistency of the system while the run-time integration of an extension is in progress. These coordination protocols are however extremely complex, especially in distributed systems [5].

With the above management architecture built on top of the Lasagne component model, the required coordination mechanisms can be kept very simple. This is a result of the strict separation between the deployment and the dynamic selection of extensions (integration $=$ deployment + selection):

During deployment of a new extension, it is only required that the wrapping commands for that extension are all correctly accepted by the component managers. This can easily be supported with a simple 2-phase commit protocol between the configuration management tool and the component managers. It is important to realize that the "acceptance of the wrapper commands" does not involve the construction of the wrapper instances nor turning the switch to actually integrate these wrapper instances into the core system.

Instead, the "turning of the switch" is under control of interceptors that dynamically select extensions by manipulating the composition policy of ongoing collaborations. The coordination mechanism that takes care of preserving the consistency while turning the switch is very simple:

- A client can only select those extensions whose extension identifiers are published by the system manager, who on his turn has already made sure before that these extensions were properly deployed into the system.

- Once an extension is selected for a client request, its consistent integration is automatically taken care of by the Lasagne component model due to the propagation of composition policies.

Inconsistent composition policies. To deal with inconsistent client-specific composition policies, the system manager has to program a deployment-specific interceptor that detects and overrides inconsistent composition policies issued by clients. This is sufficient since deploymentspecific interceptors have precedence over client-specific interceptors.

For example, the code example below shows such a deployment-specific interceptor for the dating system example of section 2.2. The interceptor first imposes access control as a mandatory extension and, secondly it filters out composition policies that apply the "leisure" and the "business" extensions at the same time, which is a conflict because these extensions implement the same concern.

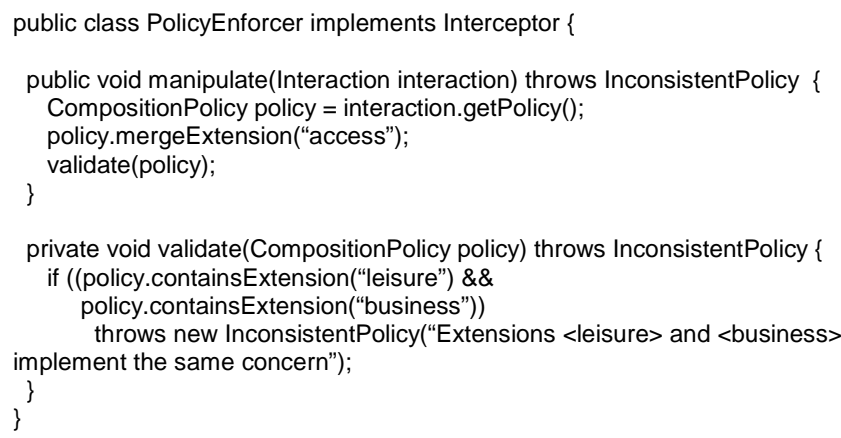

\section{Related Work}

To our knowledge, there does not exist another work that addresses the problem of customization of on-line services in the presence of simultaneous client-specific views.

The discussion on other related work is organized according to the research areas that our work touches.

\subsection{Advanced separation of concerns}

State-of-the-art separation of concerns techniques such as Aspect-oriented Programming [12], Hyperspaces [24], Mixin Layers [23], Adaptive Plug and Play Components [16] and Pluggable Composite Adapters [17] allow refinement of a core system with a crosscutting aspect, by simultaneously refining state and behavior at multiple points in the application in a non-invasive and modular way. One of the strong arguments in favor of these techniques is that they make it easier to preserve the consistency of the system. The implementation of a crosscutting extension can now be programmed in a welllocalized manner with automatic weaving/integration of aspects into the core system. These approaches however mainly operate at the class-level, limiting the integration of aspects to compile- or load-time. Lasagne on the contrary integrates crosscutting aspects at the instance-level, enabling run-time customization and client-specific customization. The price to pay for this gain of flexibility is that the Lasagne model brings several new consistency management problems of its own. However, the proposed management architecture in section 3.1 deals with these problems effectively.

Composition Filters [2] and Aspect Components [20][21] support dynamic combination of aspects on a per object interaction basis. However, these architectures do not provide the necessary coordination mechanisms to preserve consistency in the presence of dynamic 
combination of system-wide extensions on a per collaboration basis. This can however be added, since the design of these architectures are very generic. For example we implemented Lasagne on top of the Java implementation of Aspect Components (JAC) [21]. With regard to the composition filters model, incoming messages can be delegated to a meta-object that implements the necessary coordination mechanisms.

Linda Seiter et al. [22] proposed a context relation to dynamically modify a group of base classes. A context class contains several method updates for several base classes. A context object may be dynamically attached to a base object, or it may be attached to a collaboration, in which case it is implicitly attached to the set of base objects involved in that collaboration. This makes the underlying mechanism behind context relations very similar to the propagating composition policies of Lasagne. However, context relations have overriding semantics and do no allow selective combination of extensions.

Mira Mezini [15] presented the object model Rondo that does well support dynamic composition of object behavior without name collisions. However, there is no support mentioned for specifying behavior composition on a per collaboration basis. Research is however ongoing to make her co-work with Karl Lieberherr about composing collaborations [16] [17] more dynamic [9] [18].

\subsection{Dynamic software architecture}

In Regis [14] and ArchStudio [19], systems are constructed from a number of components and connectors that encapsulate the interactions between these components. The emphasis of these works is on the description, reconfiguration and evolution of the system's architecture. Connectors help to decouple components from one another and the systems use configuration languages to describe the configuration of the components. The run-time structure of the application is altered by applying a program written in the configuration language to the current architecture, thus generating a different arrangement of components and connectors. Our goal differs from these works in that we focus on customization of a system to client-specific needs, instead of coping with run-time software evolution in general. Furthermore, our customization process is based on a system-wide additive refinement of existing components, rather then replacing components with new ones and switching connectors.

Work from Andrade et al. [1] defines mathematical semantics (corresponding tool support is also implemented [7]) that integrates the notion of superimposition [11] with dynamic software architectures. Superimposition is a basic mechanism for adapting black box components (and thus is somewhat similar to the wrapper-based approach). This work takes however superimposition beyond component adaptation and adopts it as the basic structuring principle that underlies component interconnection via architectural connectors. That is to say, superimposition is taken as a mechanism via which more global interaction mechanisms can be used to coordinate the joint behavior of several components of the system. Adapting multiple components in a consistent way becomes a particular case of this coordination mechanism. As such, we feel that this work shares a common ground with Lasagne and may provide a formal framework in which we can express the semantics of Lasagne. However, this is subject of further research.

\subsection{Coordination support in adaptive systems}

As stated in section 3, performing a system-wide adaptation at run-time requires coordination mechanisms that preserve the consistency of the system while such an adaptation is in progress. In the context of distributed systems, Chen et al. [5] proposes a graceful adaptation protocol that allows adaptations to be made in a coordinated manner across hosts transparently to the application. This protocol seems more complex than the coordination mechanisms that are used with Lasagne. (look at section 3.2 for more details).

The current implementations of Lasagne seem to have a bigger run-time performance overhead though. Therefore we think that Lasagne is better suited for adaptation at the coarse-grained architectural level of a (distributed) system, while the above protocol is better suited for providing adaptability at the component implementation level.

\section{Conclusion and Discussion}

The problem that underlies this work -- the need for clients of on-line services to be able to integrate and customize these services for use in their own contexts--is a real one, and it is an important problem to resolve.

The proposed solution to this problem -- a by-request customization of the system implementing the service, where the system itself consists of a stable core and several extensions that are plugged into the core as needed -- brings some consistency management problems of its own.

We have presented the component model Lasagne that allows implementing systems that can be dynamically customized on a per client request basis. A system management architecture built on top of Lasagne deals with the mentioned consistency management problems. The design philosophy behind this management architecture is a strict separation between deployment and selection of extensions.

Unresolved problems. As stated in section 3, some kinds of extensions will inherently conflict with one another. An important issue to resolve in this matter is how does one detect and identify such conflicts? Since extensions are 
imposed dynamically in Lasagne, the only way to identify such conflicts is to wait until someone gets a bizarre result at run-time. These "bizarre results" may be context-specific (i.e., they may only occur when some set of extensions is ruming at the same time), which means that it will take the very hardest kind of debugging to track them down (essentially, debugging of concurrent systems). While one can make this argument, to some extent, about all software composition approaches, those that include more of a declarative approach have the advantage that they may be amenable to static analysis methods to identify conflicts.

\section{References}

[1] L.Andrade and J.Fiadeiro, "Coordination: The evolutionary dimension", in Proceedings of TOOLS Europe 2001, W. Pree (ed.), IEEE Computer Society Press 2001, pp. 136-147.

[2] M. Aksit, K. Wakita, J. Bosch, L. Bergmans and A. Yonezawa, "Abstracting Object-Interactions Using Composition-Filters", in Object-Based Distributed Processing, R. Guerraoui, O. Nierstrasz and M. Riveill (eds), Springer-Verlag, 1993, pp. 152-184.

[3] D. Bäumer, D. Riehle, W. Siberski and M. Wulf, "Role Object”. In Pattern Languages of Program Design 4, N. Harisson (ed.), Addison-Wesley, 2000.

[4] G. Bracha and W. Cook, "Mixin-based inheritance", in Proceeding of OOPSLA/ECOOP '90, October 1990.

[5] Wen-Ke Chen, M.A. Hiltunen, R. D. Schlichting, "Constructing Adaptive Sofware in Distributed Systems", in Proceedings of International Conference on Distributed Computing (ICDCS'2001), 2001, pp. 635-643.

[6] E. Gamma, R. Helm, R. Johnson, J. Vlissides, Design Patterns, Elements of Reusable Object-Oriented Software, Addison-Wesley, 1995, pp. 175-184.

[7] J.Gouveia, G.Koutsoukos, L.Andrade and J.Fiadeiro, "Tool support for coordination-based software evolution", in Proc. TOOLS Europe 2001, W.Pree (ed), IEEE Computer Society Press, 2001.

[8] K. Geihs, "Middleware Challenges Ahead", in IEEE Computer 34(6), June 2001.

[9] S. Hermann and M. Mezini, "PIROL, A Case-Study for Multi-Dimensional Separation of Concerns in Software Engineering Environments", in Proceedings of OOPSLA'2000, October 2000.

[10]U. Hölzle, "Integrating Independently-Developed Components in Object-Oriented Languages, in Proceedings of ECOOP' 93, 1993.

[11]S. Katz, "A Superimposition Control Construct for Distributed Systems", ACM TOPLAS 15, 1993.

[12] G. Kiczales, J. Lamping, A. Mendhekar, C. Maeda, C. V. Lopes, J. Loingtier, J. Irwan, "Aspect-Oriented Programming”, in Proceedings of ECOOP'97, June
1997.

[13] G. Kniesel, "Type-Safe Delegation for Run-Time Component Adaptation", In Proceedings of ECOOP'99, June 1999.

[14] J. Magee, N. Dulay, and J.Kramer, "Regis: A Constructive Development Environment For Distributed Programs", in Distributed Systems Engineering Journal, 1(5), 1994.

[15] M. Mezini, "Dynamic Object Evolution without Name Collisions", in Proceedings of ECOOP'97, 1997.

[16] M. Mezini and K. Lieberherr, "Adaptive Plug and Play Components for Evolutionary Software Development", in Proceedings of OOPSLA'98, 1998.

[17] M. Mezini, L. Seiter, K. Lieberherr, "Component Integration with Pluggable Composite Adapters", in Software Architectures and Component Technology: State of the Art in Research and Practice, M. Aksit (ed), 2000, Kluwer Academic Publishers.

[18] K. Ostermann, "Implementing Reusable Collaborations with Delegation Layers", First Workshop on Language Mechanisms for Programming Software Components at OOPSLA 2001.

[19]P. Oreizy, N. Medvidovic, and R.N. Taylor, "Architecture-based Run-time Software Evolution", in Proceedings of ICSE'98, 1998.

[20]R. Pawlak, L. Duchien, G. Florin. L. Martelli, L. Seinturier, "Distributed Separation of Concerns with Aspect Components", in Proceedings of TOOLS Europe'2000, IEEE press, June 2000.

[21] R. Pawlak, L. Seinturier, L. Duchien, G. Florin, "JAC: A Flexible and Efficient Solution for Aspect-Oriented Programming in Java", in Proceedings of Reflection'2001, 2001.

[22] L. Seiter, J. Palsberg, and K. Lieberherr, "Evolution of Object Behavior using Context Relations. In IEEE Transactions on Software Engineering, 24(1), 1998.

[23] Y. Smaragdakis and D. Batory, "Implementing Layered Designs with Mixin Layers", in Proceedings of ECOOP'98, 1998.

[24] P. Tarr, H. Ossher, W. Harrison, S. Sutton Jr., "N Degrees of Separation: Multi-Dimensional Separation of Concerns", in Proceedings of ICSE'99, 1999.

[25]E. Truyen, B. N. Jørgensen, W. Joosen, "Customization of Component-Based Object Request Brokers through Dynamic Configuration", in Proceedings of TOOLS Europe'2000, IEEE press, June 2000.

[26] E. Truyen, B. Vanhaute, W. Joosen, P. Verbaeten and B. N. Jørgensen, "Dynamic and Selective Combination of Extensions in Component-based Applications", in Proceedings of the 23rd International Conference on Software Engineering (ICSE'2001), 2001.

[27] Y. Wind, "The Challenge of "Customerization" in Financial Services", in Communications of the ACM, 44(16), June 2001, p. 39. 